\title{
Clicks for doughnuts
}

\author{
The ideas of topology are breaking ground in origami-based metamaterials. Experiments now show that certain \\ shapes - doughnuts included - exhibit topological bistability, and can be made to click between different \\ topologically stable states.
}

\section{Scott R. Waitukaitis}

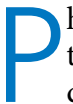
hysicists and mathematicians seem to reveal a hidden sweet tooth for doughnuts when trying to explain topology. As the classic example goes, a doughnut and a coffee cup are said to be 'topologically equivalent' because, in principle, one can be continuously deformed into the other. A doughnut and a Danish, though similarly tasty, are not equivalent because a singularity - the tear necessary to create the hole in the Danish - must be crossed before such a continuous transformation can occur. These abstract considerations of space, connectivity and conformation are the basis of topology, which is experiencing a heyday of sorts in contemporary physics.

Writing in Nature Physics, Bin Liu and colleagues ${ }^{1}$ have uncovered fascinating new implications from topology for the properties of origami-based mechanical metamaterials. Although theirs is not the first foray into topological origami ${ }^{2}$, they have taken the significant step of showing how fold vertices, the base units of origami, can exhibit 'topologically bistability'. They have also succeeded in showing that individual vertices in cleverly designed tessellations can be sequentially driven to transition, or 'click', between different topologically stable states during mechanical deformation.

As a primer to their ideas, Fig. 1a illustrates a single origami four-vertex, a point where four folds connected by four plates meet. A critical simplifying assumption in most studies is that the plates are rigid - thus rendering the four-vertex as the first $n$-vertex with a (single) continuous degree of freedom. The 'fold space' of the vertex, the region of allowed angles for the folds, is a simply connected line (Fig. 1a). Imagining that the energy of the vertex comes from torsional springs in the folds, it can be shown that generic four-vertices are guaranteed to have (at least) two energetic minima along this line ${ }^{3,4}$. This 'classical' bistability is therefore a result of multiple energy minima in the simply connected fold space of the vertex.
Liu and colleagues considered what happens when the fold space is no longer simply connected. As it turns out, one way to achieve this is to relax the simplification of rigid plates. Fully incorporating plate bending is a difficult endeavour ${ }^{5}$, but the first-order approximation is to replace bends with a finite number of 'virtual folds' (Fig. 1b). Each virtual fold enlarges the fold space by one degree of freedom, but particular combinations of base-vertex geometry and virtual fold angles can lead to geometric singularities (Fig. 1b). And much like the Danish pastry, the fold space can be torn. In particular, Liu and colleagues identified patterns that allow the space to be ripped in half-thus resulting in two separated domains instead of a single one with a hole. The consequence of this topological change on the stability landscape is that each domain is guaranteed to have at least one energy minimum, thus rendering the vertex bistable if transitions between the domains are somehow managed.

This picture is insightful, but certainly nuanced. If the fold space is no longer simply connected, then by definition transitions between the separate domains are impossible. What gives? As it turns out, it's the plates. Each additional virtual fold better approximates real plate bending, but as long as their number is finite the vertex is always left with regions between the virtual folds that can - and do - bend. A vertex at a stable state in one domain can therefore jump to another one if the energy required for inter-fold plate bending is exceeded. In the case of an infinite number of virtual folds, a continuum vertex, it can be shown that all possible configurations are simply connected ${ }^{6}$ and therefore transitions are always possible. Thus the complexities of plate bending, which the authors have more rosily referred to as 'hidden degrees of freedom? ${ }^{7}$, vitally enrich the physics. In one limit, the inclusion of a few virtual folds reveals the guiding principle of topologically disconnected domains in the fold space. At the other limit, with an infinite number of virtual folds, one is always left with a
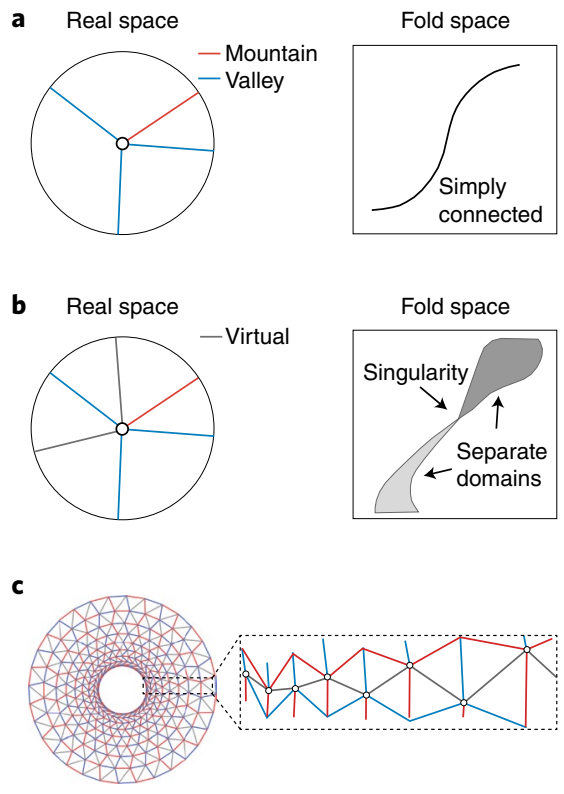

Fig. 1| Topological folding. a, A four-vertex structure consists of four folds that meet at a point. Having one degree of freedom, the 'fold space' of a four-vertex is a simply connected line, along which one or more energetic minima can exist. b. With the addition of 'virtual folds' (grey lines) to approximate plate bending, the fold space gains more degrees of freedom. For certain vertex geometries and virtual fold angles, the fold space can pass through a singularity and split into separated domains, which renders it 'topological bistable'. c, With a cleverly designed 'doughnut' tessellation, one can create a structure that exhibits a cascade of topological transitions during compression.

pathway that permits transitions between these domains so long as the penalty for bending can be exceeded.

Fascinating as these ideas are, they aren't much use unless they can be extended beyond a single vertex. Liu and colleagues did just this and revealed that the topological bistability can be programmed into cleverly designed vertex tessellations. 
Betraying their own sweet tooth, the pattern they came up with just happens to be a real-space origami doughnut (Fig. 1c). Radial strips emanating from the centre of this doughnut consist of chains of linked, Miura-ori vertices. As a strip is compressed along its length, individual vertices sequentially 'click' as they transition from one topologically stable state to the other. Crossing the threshold for each transition, the stiffness is briefly and dramatically reduced, leading to non-monotonic and even negative modulus behaviour in the composite structure.

These results open up exciting possibilities for the future of origami-based metamaterials. On the theoretical side, they establish the challenge of finding new singularities in the fold space. Liu and colleagues have shown that the fold space can be torn in half - is it also possible to tear a hole to make a fold space doughnut? Experimentally, what other kind of tricks can be played to use topology to affect the mechanical response of origami tessellations? Such questions are sure to keep physicists busy with topological folding for some time to come.
Universiteit Leiden, Leiden, the Netherlands. e-mail:waitukaitus@physics.leidenuniv.nl

Published online: 28 May 2018

https://oi.org/10.1038/s41567-018-0160-6

References

1. Liu, B. et al. Nat. Phys. https://doi.org/10.1038/s41567-018-0150-8 (2018).

2. Chen, B. G.-g et al. Phys. Rev. Lett. 116, 135501 (2016).

3. Waitukaitis, S., Menaut, R., Chen, B. G.-G. \& van Hecke, M. Phys. Rev. Lett. 114, 055503 (2015).

4. Waitukaitis, S. \& van Hecke, M. Phys. Rev. E 93, 023003 (2016).

5. Lechenault, F. \& Adda-Bedia, M. Phys. Rev. Lett. 115, 235501 (2015).

6. Streinu, I. \& Whiteley, W. in Discrete and Computational Geometry (eds Akiyama, J., Kano, M. \& Tan, X.) 161-173 (Springer, Berlin, Heidelberg, 2004).

7. Silverberg, J. L. et al. Nat. Mater. 14, 389-393 (2015).

\title{
CELL BIOPHYSICS
}

\section{Stars take centre stage}

\author{
Magnetic tweezer measurements have revealed the forces associated with a star-shaped structure responsible for \\ moving the sperm nucleus to the centre of the egg cell following fertilization.
}

\section{Carlos Garzon-Coral and Jonathon Howard}

$\mathrm{D}$ uring fertilization, after the union of sperm and egg, the male and female nuclei meet and fuse to form the zygote, also known as the one-cell embryo. But egg cells are large - those of humans and sea urchins have diameters of around $100 \mu \mathrm{m}$, and frog eggs can measure up to $1 \mathrm{~mm}$. This makes for an arduous migratory journey to the cell centre, as the nuclei themselves are no more than $10 \mu \mathrm{m}$. To meet this challenge, the nuclei make use of a star-shaped aster of microtubules growing out of a structure called the centrosome. The importance of this aster has long been recognized ${ }^{1}$, and yet the mechanics of nuclear migration remains poorly understood. Now, writing in Nature Physics, Hirokazu Tanimoto and co-workers ${ }^{2}$ have measured the force associated with the migration and centring of the microtubule aster in large cells $(\sim 90 \mu \mathrm{m})$ - providing the first estimate of the number of motor proteins involved in the process, and offering insight into the mechanism by which the motor forces are transmitted to the aster.

Thus far, only a few studies have succeeded in measuring forces associated with microtubule asters in cells ${ }^{3,4}$. Tanimoto and co-workers' success in doing so required the right organism, an outstanding instrument and a stroke of luck. Tanimoto and colleagues used the sea urchin embryo as their model system - an excellent choice owing to its large size and transparency,

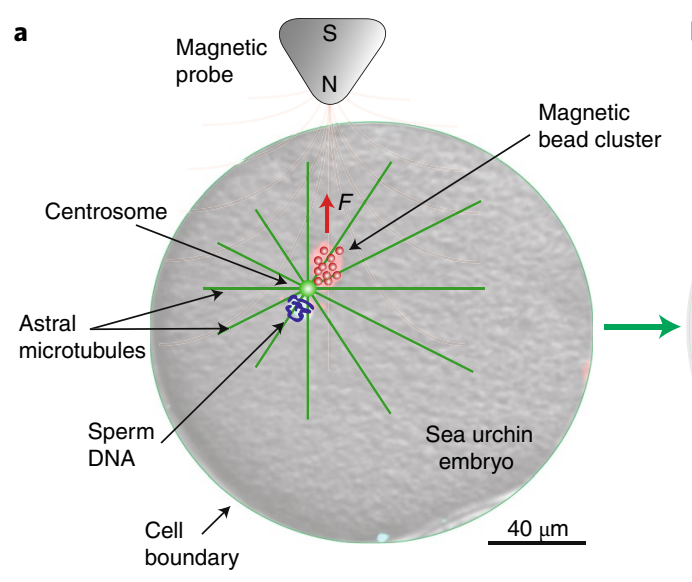

b

Fig. 1| Probing nuclear mechanics. a, A cluster of magnetic beads pull on the aster to displace it away from the cell centre. $\mathbf{b}$, Viscoelastic forces oppose the aster movement in the transverse direction, while purely viscous forces oppose motion in the radial direction.

which allowed them to inject chemicals and micrometre-sized super-paramagnetic beads into the cell. Their stroke of luck came when the beads spontaneously bound to the microtubule aster and moved to its centre. Presumably they picked up motor proteins (dyneins) that moved them centripetally along the microtubules. This self-targeting clump of magnetic beads allowed the authors to apply forces to the aster using the magnetic field gradient generated at the tip of a sharpened metal probe (Fig. 1). Forces in excess of $1,000 \mathrm{pN}$ could be applied to the aster in any direction. These forces correspond to those generated by hundreds of motor proteins, and so are ideal for probing nuclear motions.

The study found that a force of about $600 \mathrm{pN}$ pulling the beads away from the cell centre could stall the movement of the aster. A larger force could reverse the movement direction so that the aster moved away from the cell centre. Conversely, 